The Journal of $\mathbf{N}_{\text {onlinear }} \mathbf{S}_{\text {ciences and }}$ Applications http://www.tjnsa.com

\title{
B.Y. CHEN INEQUALITIES FOR BI-SLANT SUBMANIFOLDS IN GENERALIZED COMPLEX SPACE FORMS
}

\author{
S.S. SHUKLA ${ }^{1}$ AND PAWAN KUMAR RAO ${ }^{2 *}$
}

Abstract. The aim of the present paper is to study Chen inequalities for slant, bi-slant and semi-slant submanifolds in generalized complex space forms.

\section{INTRODUCTION}

In [7] B.Y. Chen recalls one of the basic problems in submanifold theory as to find simple relationships between the main extrinsic invariants and the main intrinsic invariants of a submanifold. In [5] he established a sharp inequality for the sectional curvature of a submanifold in a real space forms in terms of the scalar curvature and squared mean curvature. Afterward several geometers $[16],[20],[23]$ obtained similar inequalities for submanifolds in generalized complex space forms. Many geometers also studied contact version of above inequalities [1],[13],[15]. In this article, we establish Chen inequalities for bi-slant and semislant submanifolds in generalized complex space forms.

\section{Preliminaries}

Let $\tilde{M}$ be an almost Hermitian manifold with an almost complex structure $J$ and Riemannian metric $g$. If $J$ is integrable, i.e. the Nijenhuis tensor $[J, J]$ of $J$ vanishes, then $\tilde{M}$ is called a Hermitian manifold. The fundamental 2-form $\Omega$ of $\tilde{M}$ is defined by

$$
\Omega(X, Y)=g(X, J Y) \text {, for all, } X, Y \in T \tilde{M} .
$$

Date: Received: 16 June 2010.

* Corresponding author

(c) 2010 N.A.G.

2000 Mathematics Subject Classification. 53C40, 53C15.

Key words and phrases. Chen inequalities, slant submanifold, semi-slant submanifold, generalized complex space form. 
An almost Hermitian manifold $\tilde{M}$ is called an almost Kaehler manifold if the fundamental 2-form $\Omega$ is closed and it becomes Kaehler manifold if $\tilde{\nabla} J=0$, where $\tilde{\nabla}$ denotes the operator of covariant differentiation with respect to $g$ on $\tilde{M}$.

If an almost complex structure $J$ satisfies

$$
\left(\tilde{\nabla}_{X} J\right) Y+\left(\tilde{\nabla}_{Y} J\right) X=0
$$

for any vector fields $X$ and $Y$ on $\tilde{M}$, then the manifold is called a nearly Kaehler manifold.

A. Gray [14] introduced the notion of constant type for a nearly Kaehler manifold, which led to the definition of RK-manifolds. An RK-manifold $\tilde{M}$ is an almost Hermitian manifold for which the curvature tensor $\tilde{R}$ is $J$-invariant, i.e.

$$
\tilde{R}(J X, J Y, J Z, J W)=\tilde{R}(X, Y, Z, W),
$$

for all vector fields $X, Y, Z, W \in T \tilde{M}$.

An almost Hermitian manifold $\tilde{M}$ is said to have (pointwise) constant type if for each $p \in \tilde{M}$ and for all vector fields $X, Y, Z \in T_{p} \tilde{M}$ such that

$$
\begin{gathered}
g(X, Y)=g(X, Z)=g(X, J Y)=g(X, J Z)=0, \\
g(Y, Y)=1=g(Z, Z)
\end{gathered}
$$

we have

$$
\tilde{R}(X, Y, X, Y)-\tilde{R}(X, Y, J X, J Y)=\tilde{R}(X, Z, X, Z)-\tilde{R}(X, Z, J X, J Z) .
$$

An RK-manifold $\tilde{M}$ has (pointwise) constant type if and only if there is a differentiable function $\alpha$ on $\tilde{M}$ such that

$$
\begin{gathered}
\tilde{R}(X, Y, X, Y)-\tilde{R}(X, Y, J X, J Y)=\alpha\left\{g(X, X) g(Y, Y)-g^{2}(X, Y)\right. \\
\left.-g^{2}(X, J Y)\right\},
\end{gathered}
$$

for all vector fields $X, Y \in T \tilde{M}$.

Furthermore, $\tilde{M}$ has global constant type if $\alpha$ is constant. The function $\alpha$ is called the constant type of $\tilde{M}$. An RK-manifold of constant holomorphic sectional curvature $c$ and constant type $\alpha$ is called a generalized complex space form, denoted by $\tilde{M}(c, \alpha)$. The curvature tensor $\tilde{R}$ of $\tilde{M}(c, \alpha)$ has the following expression:

$$
\begin{aligned}
\tilde{R}(X, Y, Z, W)= & \frac{c+3 \alpha}{4}\{g(X, Z) g(Y, W)-g(X, W) g(Y, Z)\} \\
& +\frac{c-\alpha}{4}\{g(J X, Z) g(J Y, W)-g(J X, W) g(J Y, Z) \\
& +2 g(X, J Y) g(Z, J W)\},
\end{aligned}
$$

for all vector fields $X, Y, Z, W \in T \tilde{M}$. 
If $c=\alpha$, then $\tilde{M}(c, \alpha)$ is a space of constant curvature. A complex space form $\tilde{M}(c)$ (i.e., a Kaehler manifold of constant holomorphic sectional curvature $c$ ) belongs to the class of almost Hermitian manifold $\tilde{M}(c, \alpha)$ (with constant type zero).

Let $M$ be a Riemannian manifold and $K(\pi)$ the sectional curvature of $M$ associated with a plane section $\pi \subset T_{p} M, p \in M$.

For any orthonormal basis $\left\{e_{1}, \ldots ., e_{n}\right\}$ of the tangent space $T_{p} M$, the scalar curvature $\tau$ at $p$ is defined by

$$
\tau(p)=\sum_{i<j} K\left(e_{i} \wedge e_{j}\right)
$$

We denote by

$$
(\inf K)(p)=\inf \left\{K(\pi): \pi \subset T_{p} M, \operatorname{dim} \pi=2\right\} .
$$

The first Chen invariant $\delta_{M}(p)$ is given by

$$
\delta_{M}(p)=\tau(p)-(\inf K)(p) .
$$

Let $L$ be a subspace of $T_{p} M$ of dimension $k \geq 2$ and $\left\{e_{1}, \ldots . ., e_{k}\right\}$ an orthonormal basis of $L$. Define $\tau(L)$ be the scalar curvature of the $k$-plane section $L$ by

$$
\tau(L)=\sum_{i<j} K\left(e_{i} \wedge e_{j}\right), i, j=1, \ldots ., k .
$$

Given an orthonormal basis $\left\{e_{1}, \ldots . ., e_{n}\right\}$ of the tangent space $T_{p} M$, we denote by $\tau_{1 \ldots . . k}$ the scalar curvature of $k$-plane section spanned by $e_{1}, \ldots \ldots, e_{k}$. The scalar curvature $\tau(p)$ of $M$ at $p$ is the scalar curvature of the tangent space of $M$ at $p$. If $L$ is a 2-plane section, then $\tau(L)$ reduces to the sectional curvature $K(L)$ of the plane section $L$. If $K(\pi)$ is the sectional curvature of $M$ for a plane section $\pi$ in $T_{p} M, p \in M$, then scalar curvature $\tau(p)$ at $p$ is given by

$$
\tau(p)=\sum_{i<j} K_{i j}
$$

where $\left\{e_{1}, \ldots ., e_{n}\right\}$ is an orthonormal basis for $T_{p} M$ and $K_{i j}$ is the sectional curvature of the plane section spanned by $e_{i}$ and $e_{j}$ at $p \in M$.

We recall the following Lemma of Chen [6].

Lemma 2.1. Let $n \geq 2$ and $a_{1}, \ldots . ., a_{n}, b$ be $(n+1)$-real numbers, such that

$$
\left(\sum_{i=1}^{n} a_{i}\right)^{2}=(n-1)\left(\sum_{i=1}^{n} a_{i}^{2}+b\right)
$$

Then $2 a_{1} a_{2} \geq b$ with equality holding if and only if

$$
a_{1}+a_{2}=a_{3}=\ldots \ldots=a_{n} .
$$


Let $M$ be an n-dimensional submanifold of a $2 \mathrm{~m}$-dimensional generalized complex space form $\tilde{M}(c, \alpha)$ and we denote by $h, \nabla$ and $\nabla^{\perp}$ the second fundamental form of $M$, the induced connection on $M$ and the normal bundle $T^{\perp} M$. Then, the Gauss and Weingarten formulae are given respectively

$$
\tilde{\nabla}_{X} Y=\nabla_{X} Y+h(X, Y)
$$

and

$$
\tilde{\nabla}_{X} V=-A_{V} X+\nabla_{X}^{\perp} V
$$

for all vector fields $X, Y$ tangent to $M$ and vector field $V$ normal to $M$, where $A_{V}$ is the shape operator in the direction of $V$. The second fundamental form and the shape operator are related by

$$
g(h(X, Y), V)=g\left(A_{V} X, Y\right) .
$$

Let $R$ be the Riemannian curvature tensor of $M$, then the equation of Gauss is given by,

$$
\begin{gathered}
\tilde{R}(X, Y, Z, W)=R(X, Y, Z, W)+g(h(X, W), h(Y, Z)) \\
-g(h(X, Z), h(Y, W)),
\end{gathered}
$$

for any vector fields $X, Y, Z, W$ tangent to $M$.

Let $p \in M$ and $\left\{e_{1}, \ldots ., e_{n}\right\}$ an orthonormal basis of the tangent space $T_{p} M$. We denote by $H(p)$ the mean curvature vector at $p$, that is

$$
H(p)=\frac{1}{n} \sum_{i=1}^{n} h\left(e_{i}, e_{i}\right)
$$

Also, we set

$$
h_{i j}^{r}=g\left(h\left(e_{i}, e_{j}\right), e_{r}\right), i, j \in\{1, \ldots \ldots \ldots \ldots, n\}, r \in\{n+1, \ldots . ., 2 m\},
$$

and

$$
\|h\|^{2}=\sum_{i, j=1}^{n} g\left(h\left(e_{i}, e_{j}\right), h\left(e_{i}, e_{j}\right)\right) .
$$

For any $p \in M$ and $X \in T_{p} M$, we put

$$
J X=P X+F X,
$$

where $P X$ and $F X$ are the tangential and normal components of $J X$ respectively.

Let us denote

$$
\|P\|^{2}=\sum_{i, j=1}^{n} g^{2}\left(P e_{i}, e_{j}\right)
$$


Now, we recall that for a submanifold $M$ in a Riemannian manifold, the relative null space of $M$ at a point $p$ is defined by

$$
N_{p}=\left\{X \in T_{p} M \mid h(X, Y)=0, \text { for all } Y \in T_{p} M\right\} .
$$

Definition(2.1)[2]. A differential distribution $D$ on $M$ is called a slant distribution if for each $p \in M$ and each non-zero vector $X \in D_{p}$, the angle $\theta_{D}(X)$ between $J X$ and the vector subspace $D_{p}$ is constant, which is independent of the choice of $p \in M$ and $X \in D_{p}$. In this case, the constant angle $\theta_{D}$ is called the slant angle of the distribution $D$.

Definition(2.2)[2]. A submanifold $M$ is said to be a slant submanifold if for any $p \in M$ and $X \in T_{p} M$, the angle between $J X$ and $T_{p} M$ is constant, i.e., it does not depend on the choice of $p \in M$ and $X \in T_{p} M$. The angle $\theta \in\left[0, \frac{\pi}{2}\right]$ is called the slant angle of $M$ in $\tilde{M}$.

Invariant and anti-invariant submanifolds are slant submanifolds with slant angle $\theta=0$ and $\theta=\frac{\pi}{2}$, respectively. A slant submanifold which is neither invariant nor anti-invariant is called a proper slant submanifold.

Definition(2.3)[3]. A submanifold $M$ is called a bi-slant submanifold of $\tilde{M}$ if there exist two orthogonal distributions $D_{1}$ and $D_{2}$ on $M$, such that

(i) $T M$ admits the orthogonal direct decomposition $T M=D_{1} \oplus D_{2}$,

(ii) for any $i=1,2, D_{i}$ is slant distribution with slant angle $\theta_{i}$.

On the other hand, CR-submanifolds of $\tilde{M}$ are bi-slant submanifolds with $\theta_{1}=0$ and $\theta_{2}=\frac{\pi}{2}$.

Let $2 d_{1}=\operatorname{dim} D_{1}$ and $2 d_{2}=\operatorname{dim} D_{2}$.

If either $d_{1}$ or $d_{2}$ vanishes, the bi-slant submanifold is a slant submanifold. Thus, slant submanifolds are particular cases of bi-slant submanifolds.

Definition(2.4)[3]. A submanifold $M$ is said to be a semi-slant submanifold of $\tilde{M}$ if there exist two orthogonal distributions $D_{1}$ and $D_{2}$ on $M$, such that

(i) $T M$ admits the orthogonal direct decomposition $T M=D_{1} \oplus D_{2}$,

(ii) the distribution $D_{1}$ is an invariant distribution, that is, $J\left(D_{1}\right)=D_{1}$,

(iii) the distribution $D_{2}$ is slant with angle $\theta \neq 0$.

The invariant distribution of a semi-slant submanifold is a slant distribution with zero slant angle. Thus, it is obvious that, semi-slant submanifolds are particular cases of bi-slant submanifolds. However if $2 d_{1}=\operatorname{dim} D_{1}$ and $2 d_{2}=\operatorname{dim} D_{2}$

(a) $d_{2}=0$, then $M$ is an invariant submanifold.

(b) $d_{1}=0$ and $\theta=\frac{\pi}{2}$, then $M$ is an anti-invariant submanifold.

(c) $d_{1}=0$ and $\theta \neq \frac{\pi}{2}$, then $M$ is a proper slant submanifold, with slant angle $\theta$.

A semi-slant submanifold is proper if $d_{1} d_{2} \neq 0$ and $\theta \neq \frac{\pi}{2}$. 


\section{B.Y. Chen inequalities}

In this section, we establish Chen inequalities for proper bi-slant submanifolds in a generalized complex space form. We consider a plane section $\pi$ invariant by $P$ and denote $\operatorname{dim} D_{1}=2 d_{1}$ and $\operatorname{dim} D_{2}=2 d_{2}$.

Theorem 3.1. Let $M$ be an n-dimensional proper bi-slant submanifold of a 2m-dimensional generalized complex space form $\tilde{M}(c, \alpha)$. Then

(I) For any plane section $\pi$ invariant by $P$ and tangent to $D_{1}$,

$$
\begin{aligned}
\delta_{M} \leq \frac{n-2}{2} & \left\{\frac{n^{2}}{n-1}\|H\|^{2}+\frac{c+3 \alpha}{4}(n+1)\right\} \\
& +\frac{(c-\alpha)}{4}\left\{3\left(d_{1}-1\right) \cos ^{2} \theta_{1}+3 d_{2} \cos ^{2} \theta_{2}\right\}
\end{aligned}
$$

and

(II) For any plane section $\pi$ invariant by $P$ and tangent to $D_{2}$,

$$
\begin{aligned}
\delta_{M} \leq \frac{n-2}{2} & \left\{\frac{n^{2}}{n-1}\|H\|^{2}+\frac{c+3 \alpha}{4}(n+1)\right\} \\
& +\frac{c-\alpha}{4}\left\{3 d_{1} \cos ^{2} \theta_{1}+3\left(d_{2}-1\right) \cos ^{2} \theta_{2}\right\} .
\end{aligned}
$$

The equality case of inequalities (3.1) and (3.2) hold at a point $p \in M$ if and only if there exists an orthonormal basis $\left\{e_{1}, e_{2}, \ldots ., e_{n}\right\}$ of $T_{p} M$ and an orthonormal basis $\left\{e_{n+1}, \ldots . ., e_{2 m}\right\}$ of $T_{p}^{\perp} M$ such that the shape operators of $M$ in $\tilde{M}(c, \alpha)$ at $p$ have the following forms:

$$
\begin{aligned}
& A_{n+1}=\left(\begin{array}{ccccc}
a & 0 & 0 & \ldots . & 0 \\
0 & b & 0 & \ldots . & 0 \\
0 & 0 & \mu & \ldots . & 0 \\
. . & . . & . . & \ldots . & \\
0 & 0 & 0 & \ldots . & \mu
\end{array}\right), \quad a+b=\mu, \\
& A_{r}=\left(\begin{array}{ccccc}
h_{11}^{r} & h_{12}^{r} & 0 & \ldots . & 0 \\
h_{12}^{r} & -h_{11}^{r} & 0 & \ldots . & 0 \\
0 & 0 & 0 & \ldots . & 0 \\
. . & . & . . & \ldots . & \\
0 & 0 & 0 & \ldots . . & 0
\end{array}\right)
\end{aligned}
$$

where

$$
\begin{aligned}
& A_{r}=A_{e_{r}}, r=n+1, \ldots ., 2 m . \\
& h_{i j}^{r}=g\left(h\left(e_{i}, e_{j}\right), e_{r}\right), r=n+1, \ldots ., 2 m .
\end{aligned}
$$

Proof. The Gauss equation for the submanifold $M$ is given by

$$
\begin{gathered}
\tilde{R}(X, Y, Z, W)=R(X, Y, Z, W)+g(h(X, W), h(Y, Z)) \\
-g(h(X, Z), h(Y, W)),
\end{gathered}
$$


for all vector fields $X, Y, Z, W \in T M$, where $\tilde{R}, R$ denote the curvature tensors of $\tilde{M}(c, \alpha)$ and $M$ respectively.

The curvature tensor $\tilde{R}$ of $\tilde{M}(c, \alpha)$ has the following expression [20]:

$$
\begin{aligned}
\tilde{R}(X, Y, Z, W)=\frac{c+3 \alpha}{4} & \{g(X, Z) g(Y, W)-g(X, W) g(Y, Z)\} \\
& +\frac{c-\alpha}{4}\{g(J X, Z) g(J Y, W)-g(J X, W) g(J Y, Z) \\
& +2 g(X, J Y) g(Z, J W)\},
\end{aligned}
$$

for any vector fields $X, Y, Z, W \in T M$.

Let $p \in M$, we choose an orthonormal basis $\left\{e_{1}, e_{2}, \ldots ., e_{n}\right\}$ of $T_{p} M$ and an orthonormal basis $\left\{e_{n+1}, \ldots ., e_{2 m}\right\}$ of $T_{p}^{\perp} M$. By substituting $X=Z=e_{i}, Y=$ $W=e_{j}$ in equation (3.8), we have

$$
\begin{aligned}
\tilde{R}\left(e_{i}, e_{j}, e_{i}, e_{j}\right)= & \frac{c+3 \alpha}{4}\left\{n^{2}-n\right\} \\
& +\frac{c-\alpha}{4}\left\{-g\left(J e_{i}, e_{j}\right) g\left(J e_{j}, e_{i}\right)+2 g\left(e_{i}, J e_{j}\right) g\left(e_{i}, J e_{j}\right)\right. \\
= & \frac{c+3 \alpha}{4}\left\{n^{2}-n\right\}+\frac{c-\alpha}{4}\left\{3 \sum_{i, j=1}^{n} g^{2}\left(J e_{i}, e_{j}\right)\right\} .
\end{aligned}
$$

Let $M$ be a proper bi-slant submanifold of $\tilde{M}(c, \alpha)$ and $\operatorname{dim} M=n=2 d_{1}+2 d_{2}$. We consider an adapted bi-slant orthonormal frames

$$
\begin{aligned}
& e_{1}, e_{2}=\frac{1}{\cos \theta_{1}} P e_{1}, \ldots \ldots, e_{2 d_{1}-1}, e_{2 d_{1}}=\frac{1}{\cos \theta_{1}} P e_{2 d_{1}-1}, \\
& e_{2 d_{1}+1}, e_{2 d_{1}+2}=\frac{1}{\cos \theta_{2}} P e_{2 d_{1}+1}
\end{aligned}
$$

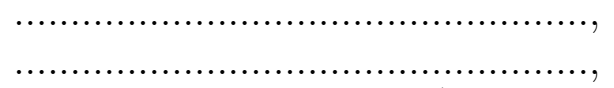

$$
\begin{aligned}
& e_{2 d_{1}+2 d_{2}-1}, e_{2 d_{1}+2 d_{2}}=\frac{1}{\cos \theta_{2}} P e_{2 d_{1}+2 d_{2}-1} .
\end{aligned}
$$

Obviously, we have

$$
\begin{aligned}
g^{2}\left(J e_{i}, e_{i+1}\right)= & \cos ^{2} \theta_{1}, \text { for } i \in\left\{1, \ldots ., 2 d_{1}-1\right\} \text { and } \\
& =\cos ^{2} \theta_{2}, \text { for } i \in\left\{2 d_{1}+1, \ldots ., 2 d_{1}+2 d_{2}-1\right\} .
\end{aligned}
$$

Then, we have

$$
\sum_{i, j=1}^{n} g^{2}\left(J e_{i}, e_{j}\right)=2\left(d_{1} \cos ^{2} \theta_{1}+d_{2} \cos ^{2} \theta_{2}\right)
$$

Substituting (3.12) into (3.9), we have

$$
\tilde{R}\left(e_{i}, e_{j}, e_{i}, e_{j}\right)=\frac{c+3 \alpha}{4}\left\{n^{2}-n\right\}+\frac{c-\alpha}{4}\left\{6\left(d_{1} \cos ^{2} \theta_{1}+d_{2} \cos ^{2} \theta_{2}\right)\right\} .
$$

The equation (3.7) gives

$$
\tilde{R}\left(e_{i}, e_{j}, e_{i}, e_{j}\right)=2 \tau+\|h\|^{2}-n^{2}\|H\|^{2} .
$$

By using equations (3.13) and (3.14), we get 


$$
2 \tau=n^{2}\|H\|^{2}-\|h\|^{2}+\frac{c+3 \alpha}{4}\{n(n-1)\}+\frac{c-\alpha}{4}\left\{6\left(d_{1} \cos ^{2} \theta_{1}+d_{2} \cos ^{2} \theta_{2}\right)\right\} .
$$

If we set $\left.\left.d_{2} \cos ^{2} \theta_{2}\right)\right\}$,

$$
\epsilon=2 \tau-\frac{n^{2}}{n-1}(n-2)\|H\|^{2}-\frac{c+3 \alpha}{4}\{n(n-1)\}-\frac{c-\alpha}{4}\left\{6 \left(d_{1} \cos ^{2} \theta_{1}+\right.\right.
$$

in equation (3.15), we get

$$
n^{2}\|H\|^{2}=(n-1)\left(\epsilon+\|h\|^{2}\right) .
$$

Let $p \in M, \pi \subset T_{p} M, \operatorname{dim} \pi=2$ and $\pi$ invariant by $P$.

Now, we consider two cases:

Case (a): The plane section $\pi$ is tangent to $D_{1}$.

We may assume that $\pi=s p\left\{e_{1}, e_{2}\right\}$. We choose $e_{n+1}=\frac{H}{\|H\|}$.

From the equation (3.17) becomes,

$$
\left(\sum_{i=1}^{n} h_{i i}^{n+1}\right)^{2}=(n-1)\left\{\sum_{r=n+1}^{2 m} \sum_{i, j=1}^{n}\left(h_{i j}^{r}\right)^{2}+\epsilon\right\} .
$$

The above equation implies

$$
\left(\sum_{i=1}^{n} h_{i i}^{n+1}\right)^{2}=(n-1)\left\{\sum_{i=1}^{n}\left(h_{i i}^{n+1}\right)^{2}+\sum_{i \neq j}\left(h_{i j}^{n+1}\right)^{2}+\sum_{r=n+2}^{2 m} \sum_{i, j=1}^{n}\left(h_{i j}^{r}\right)^{2}+\epsilon\right\} .
$$

Using the Lemma (2.1) and equation (3.19), we obtain

$$
2 h_{11}^{n+1} h_{22}^{n+1} \geq \sum_{i \neq j}\left(h_{i j}^{n+1}\right)^{2}+\sum_{r=n+2}^{2 m} \sum_{i, j=1}^{n}\left(h_{i j}^{r}\right)^{2}+\epsilon .
$$

From the Gauss equation for $X=Z=e_{1}$ and $Y=W=e_{2}$, we get

$$
\begin{aligned}
K(\pi)= & \frac{c+3 \alpha}{4}+3 \frac{c-\alpha}{4} \cos ^{2} \theta_{1}+\sum_{r=n+1}^{2 m}\left[h_{11}^{r} h_{22}^{r}-\left(h_{12}^{r}\right)^{2}\right] \\
\geq & \frac{c+3 \alpha}{4}+3 \frac{c-\alpha}{4} \cos ^{2} \theta_{1}+\frac{1}{2}\left[\sum_{i \neq j}\left(h_{i j}^{n+1}\right)^{2}+\sum_{r=n+2}^{2 m} \sum_{i, j=1}^{n}\left(h_{i j}^{r}\right)^{2}+\epsilon\right] \\
& +\sum_{r=n+2}^{2 m} h_{11}^{r} h_{22}^{r}-\sum_{r=n+1}^{2 m}\left(h_{12}^{r}\right)^{2} \\
= & \frac{c+3 \alpha}{4}+3 \frac{c-\alpha}{4} \cos ^{2} \theta_{1}+\frac{1}{2} \sum_{i \neq j}\left(h_{i j}^{n+1}\right)^{2}+\frac{1}{2} \sum_{r=n+2}^{2 m} \sum_{i, j>2}\left(h_{i j}^{r}\right)^{2} \\
& +\frac{1}{2} \sum_{r=n+2}^{2 m}\left(h_{11}^{r}+h_{22}^{r}\right)^{2}+\sum_{j>2}\left[\left(h_{1 j}^{n+1}\right)^{2}+\left(h_{2 j}^{n+1}\right)^{2}\right]+\frac{\epsilon}{2} \\
\geq & \frac{c+3 \alpha}{4}+3 \frac{c-\alpha}{4} \cos ^{2} \theta_{1}+\frac{\epsilon}{2} .
\end{aligned}
$$


From the equations (3.16), (3.21) and (2.9), it follows that

$$
\begin{aligned}
\inf K \geq & \frac{c+3 \alpha}{4}+3 \frac{c-\alpha}{4} \cos ^{2} \theta_{1}+\tau-\frac{n^{2}}{2(n-1)}(n-2)\|H\|^{2} \\
& \quad-\frac{c+3 \alpha}{8}\{n(n-1)\}-\frac{c-\alpha}{8}\left\{6\left(d_{1} \cos ^{2} \theta_{1}+d_{2} \cos ^{2} \theta_{2}\right)\right\} .
\end{aligned}
$$

From the equations (3.22) and (2.10), we get

$$
\begin{aligned}
\delta_{M} \leq & \frac{n-2}{2}\left\{\frac{n^{2}}{n-1}\|H\|^{2}+\frac{c+3 \alpha}{4}(n+1)\right\} \\
& +\frac{c-\alpha}{4}\left\{3\left(d_{1}-1\right) \cos ^{2} \theta_{1}+3 d_{2} \cos ^{2} \theta_{2}\right\},
\end{aligned}
$$

where $\delta_{M}$ is Chen invariant. This proves the inequality (3.1).

Case (b): The plane section $\pi$ is tangent to $D_{2}$.

From the equation (3.17), we have

$$
\left(\sum_{i=1}^{n} h_{i i}^{n+1}\right)^{2}=(n-1)\left\{\sum_{r=n+1}^{2 m} \sum_{i, j=1}^{n}\left(h_{i j}^{r}\right)^{2}+\epsilon\right\} .
$$

The above equation implies

$$
\left(\sum_{i=1}^{n} h_{i i}^{n+1}\right)^{2}=(n-1)\left\{\sum_{i=1}^{n}\left(h_{i i}^{n+1}\right)^{2}+\sum_{i \neq j}\left(h_{i j}^{n+1}\right)^{2}+\sum_{r=n+2}^{2 m} \sum_{i, j=1}^{n}\left(h_{i j}^{r}\right)^{2}+\epsilon\right\} .
$$

Using the Lemma (2.1) and equation (3.24), we obtain

$$
2 h_{11}^{n+1} h_{22}^{n+1} \geq \sum_{i \neq j}\left(h_{i j}^{n+1}\right)^{2}+\sum_{r=n+2}^{2 m} \sum_{i, j=1}^{n}\left(h_{i j}^{r}\right)^{2}+\epsilon .
$$

From the Gauss equation for $X=Z=e_{1}$ and $Y=W=e_{2}$, we get

$$
\begin{aligned}
& K(\pi)= \frac{c+3 \alpha}{4}+3 \frac{c-\alpha}{4} \cos ^{2} \theta_{2}+\sum_{r=n+1}^{2 m}\left[h_{11}^{r} h_{22}^{r}-\left(h_{12}^{r}\right)^{2}\right] \\
& \geq \frac{c+3 \alpha}{4}+3 \frac{c-\alpha}{4} \cos ^{2} \theta_{2}+\frac{1}{2}\left[\sum_{i \neq j}\left(h_{i j}^{n+1}\right)^{2}+\sum_{r=n+2}^{2 m} \sum_{i, j=1}^{n}\left(h_{i j}^{r}\right)^{2}+\epsilon\right] \\
& \quad+\sum_{r=n+2}^{2 m} h_{11}^{r} h_{22}^{r}-\sum_{r=n+1}^{2 m}\left(h_{12}^{r}\right)^{2} \\
&=\frac{c+3 \alpha}{4}+3 \frac{c-\alpha}{4} \cos ^{2} \theta_{2}+\frac{1}{2} \sum_{i \neq j}\left(h_{i j}^{n+1}\right)^{2}+\frac{1}{2} \sum_{r=n+2}^{2 m} \sum_{i, j>2}^{n}\left(h_{i j}^{r}\right)^{2} \\
& \quad+\frac{1}{2} \sum_{r=n+2}^{2 m}\left(h_{11}^{r}+h_{22}^{r}\right)^{2}+\sum_{j>2}\left[\left(h_{1 j}^{n+1}\right)^{2}+\left(h_{2 j}^{n+1}\right)^{2}\right]+\frac{\epsilon}{2} \\
& \geq \frac{c+3 \alpha}{4}+3 \frac{c-\alpha}{4} \cos ^{2} \theta_{2}+\frac{\epsilon}{2} .
\end{aligned}
$$

From the relations (3.16), (3.26) and (2.9), it follows that 


$$
\begin{aligned}
\inf K \geq \frac{c+3 \alpha}{4}+3 \frac{c-\alpha}{4} \cos ^{2} \theta_{2}+\tau-\frac{n^{2}}{2(n-1)}(n-2)\|H\|^{2} \\
\quad-\frac{c+3 \alpha}{8}\{n(n-1)\}-\frac{c-\alpha}{8}\left\{6\left(d_{1} \cos ^{2} \theta_{1}+d_{2} \cos ^{2} \theta_{2}\right)\right\} .
\end{aligned}
$$

From the equations (3.27) and (2.10), we get

$$
\begin{aligned}
\delta_{M} \leq \frac{n-2}{2} & \left\{\frac{n^{2}}{n-1}\|H\|^{2}+\frac{c+3 \alpha}{4}(n+1)\right\}, \\
& +\frac{c-\alpha}{4}\left\{3 d_{1} \cos ^{2} \theta_{1}+3\left(d_{2}-1\right) \cos ^{2} \theta_{2}\right\} .
\end{aligned}
$$

This proves the inequality (3.2).

The equality case at a point $p$ holds, if and only if equality holds in each of inequalities (3.20), (3.23) and (3.28) and Lemma (2.1). So we have

$$
\begin{aligned}
& h_{i j}^{n+1}=0, \forall i \neq j, i, j>2, \\
& h_{i j}^{r}=0, \forall i \neq j, i, j>2, r=n+1, \ldots . ., 2 m, \\
& h_{11}^{r}+h_{22}^{r}=0, \forall r=n+2, \ldots \ldots, 2 m, \\
& h_{1 j}^{n+1}=h_{2 j}^{n+1}=0, \forall j>2, \\
& h_{11}^{n+1}+h_{2}^{n+1}=h_{33}^{n+1}=\ldots \ldots=h_{n n}^{n+1} .
\end{aligned}
$$

We may choose $\left\{e_{1}, e_{2}\right\}$ such that $h_{12}^{n+1}=0$ and we denote by $a=h_{11}, b=$ $h_{22}^{r}, \mu=h_{33}^{n+1}=\ldots \ldots .=h_{n n}^{n+1}$. Then the shape operators take the desired forms.

Now, we can state the following:

Corollary 3.2. Let $M$ be an n-dimensional proper semi-slant submanifold of a 2m-dimensional generalized complex space form $\tilde{M}(c, \alpha)$. Then

(I) For any plane section $\pi$ invariant by $P$ and tangent to $D_{1}$,

$$
\begin{aligned}
\delta_{M} \leq \frac{n-2}{2} & \left\{\frac{n^{2}}{n-1}\|H\|^{2}+\frac{c+3 \alpha}{4}(n+1)\right\} \\
& +\frac{(c-\alpha)}{4}\left\{3\left(d_{1}-1\right)+3 d_{2} \cos ^{2} \theta\right\}
\end{aligned}
$$

and

(II) For any plane section $\pi$ invariant by $P$ and tangent to $D_{2}$,

$$
\begin{aligned}
\delta_{M} \leq \frac{n-2}{2} & \left\{\frac{n^{2}}{n-1}\|H\|^{2}+\frac{c+3 \alpha}{4}(n+1)\right\} \\
& +\frac{c-\alpha}{4}\left\{3 d_{1}+3\left(d_{2}-1\right) \cos ^{2} \theta\right\} .
\end{aligned}
$$

The equality case of inequalities (3.29) and (3.30) holds at a point $p \in M$ if and only if there exists an orthonormal basis $\left\{e_{1}, e_{2}, \ldots . ., e_{n}\right\}$ of $T_{p} M$ and an orthonormal basis $\left\{e_{n+1}, \ldots . ., e_{2 m}\right\}$ of $T_{p}^{\perp} M$ such that the shape operators of $M$ in $\tilde{M}(c, \alpha)$ at $p$ have the forms (3.3) and (3.4). 
Corollary 3.3. Let $M$ be an $n$-dimensional $\theta$-slant submanifold of a $2 m$-dimensional generalized complex space form $\tilde{M}(c, \alpha)$. Then

$$
\delta_{M} \leq \frac{n-2}{2}\left\{\frac{n^{2}}{n-1}\|H\|^{2}+\frac{c+3 \alpha}{4}(n+1)+3 \frac{c-\alpha}{4} \cos ^{2} \theta\right\} .
$$

The equality case of the inequality (3.31) holds at a point $p \in M$ if and only if there exists an orthonormal basis $\left\{e_{1}, \ldots . ., e_{n}\right\}$ of $T_{p} M$ and an orthonormal basis $\left\{e_{n+1}, \ldots . ., e_{2 m}\right\}$ of $T_{p}^{\perp} M$ such that the shape operators of $M$ in $\tilde{M}(c, \alpha)$ at $p$ have the forms (3.3) and (3.4).

Corollary 3.4. Let $M$ be an $n$-dimensional invariant submanifold of a $2 m$ dimensional generalized complex space form $\tilde{M}(c, \alpha)$. Then

$$
\delta_{M} \leq \frac{n-2}{2}\left\{\frac{n^{2}}{n-1}\|H\|^{2}+\frac{c+3 \alpha}{4}(n+1)+3 \frac{c-\alpha}{4}\right\} .
$$

The equality case of the inequality (3.32) holds at a point $p \in M$ if and only if there exists an orthonormal basis $\left\{e_{1}, \ldots ., e_{n}\right\}$ of $T_{p} M$ and an orthonormal basis $\left\{e_{n+1}, \ldots ., e_{2 m}\right\}$ of $T_{p}^{\perp} M$ such that the shape operators of $M$ in $\tilde{M}(c, \alpha)$ at $p$ have the forms (3.3) and (3.4).

Corollary 3.5. Let $M$ be an $n$-dimensional anti-invariant submanifold of a $2 m$ dimensional generalized complex space form $\tilde{M}(c, \alpha)$. Then

$$
\delta_{M} \leq \frac{n-2}{2}\left\{\frac{n^{2}}{n-1}\|H\|^{2}+\frac{c+3 \alpha}{4}(n+1)\right\} .
$$

The equality case of the inequality (3.33) holds at a point $p \in M$ if and only if there exists an orthonormal basis $\left\{e_{1}, \ldots . ., e_{n}\right\}$ of $T_{p} M$ and an orthonormal basis $\left\{e_{n+1}, \ldots . ., e_{2 m}\right\}$ of $T_{p}^{\perp} M$ such that the shape operators of $M$ in $\tilde{M}(c, \alpha)$ at $p$ have the forms (3.3) and (3.4).

Acknowledgements: The authors are thankful to the referee towards the improvement of manuscript.

\section{REFERENCES}

[1] N. Aktan, M.Z. Sarikaya, E. Ozusaglam, B.Y. Chen's inequality for semislant submanifolds in T-space forms, Balkan J. Geom. Appl., 13(1) (2008), 1-10.

[2] J.L. Cabrerizo, A. Carriazo, L.M. Fernandez, M. Fernandez, Slant submanifolds in sasakian manifolds, Glasgow Math. J., 42(1) (2000), 125-138.

[3] J.L. Cabrerizo, A. Carriazo, L.M. Fernandez, M. Fernandez, Semi-slant submanifolds of a Sasakian manifold, Geometriae Dedicata, 78(2) (1999), 183-199.

[4] B.Y. Chen, Geometry of slant submanifols, K.U. Leuven, 1990.

[5] B.Y. Chen, Some pinching and classification theorems for minimal submanifolds, Arch. Math., 60 (1993), 568-578.

[6] B.Y. Chen, Some new obstructions to minimal and Lagrangian isometric immersions, Japan J. Math. (N.S.), 26 (2000), 105-127.

[7] B.Y. Chen, Mean curvature and shape operator of isometric immersions in real space forms, Glasgow Math. J., 38 (1996), 87-97.

[8] B.Y. Chen, Relations between Ricci curvature and shape operator for submanifolds with arbitrary co-dimensions, Glasgow Math. J., 41 (1999), 33-41. 
[9] B.Y. Chen, Slant immersions, Bull. Aus. Math. Soc., 41 (1990), 135-147.

[10] B.Y. Chen, Y. Tazawa, Slant submanifolds in complex Euclidean spaces, Tokyo J. Math., 14 (1991), 101-120.

[11] B.Y. Chen, L. Vrancken, Existence and Uniqueness theorem for slant immersions and its applications, Results Math., 31 (1997), 28-39.

[12] B.Y. Chen, A general inequality for Kaehlerian slant submanifolds and related results, Geometriae Dedicata, 85 (2001), 253-271.

[13] D. Cioroboiu, B.Y. Chen inequalities for semi-slant submanifolds in Sasakian space forms, IJMMS, 27 (2003), 1731-1738.

[14] A. Gray, Nearly Kaehlerian manifolds, J. Diff. Geometry, 4(1970), 283-309.

[15] R.S. Gupta, I. Ahmed, S.M.K. Haider, B.Y. Chen's inequality and its applications to slant immersions into Kenmotsu manifolds, Kyungpook Math. J., 44 (2004), 101-110.

[16] J.S. Kim, Y.M. Song, M.M. Tripathi, B.Y. Chen inequalities for submanifolds in generalized complex space forms, Bull. Korean Math. Soc., 40(3) (2003), 411-423.

[17] J.S. Kim, Y.M. Song, M.M. Tripathi, Shape operator for slant submanifolds in generalized complex space forms, Indian J. Pure appl. Math., 34(8) (2003), 1153-1163.

[18] K. Matsumoto, I. Mihai, A. Oiaga, Shape operator $A_{H}$ for slant submanifolds in complex space forms, Bull. Yamagata Univ., 14 (2000), 169-177.

[19] A. Mihai, t Shape operator $A_{H}$ for slant submanifolds in generalized complex space forms, Turk. J. Math., 27 (2003), 509-523.

[20] A. Mihai, B.Y. Chen inequalities for slant submanifolds in generalized complex space forms, Radovi Mathematicki, 12 (2004), 215-231.

[21] I. Mihai, R. Rosca, L. Veratraelen, Some aspects of the Differential Geometry of vector fields, PADGF2, K.U. Leuven, Brussels, 1996

[22] A. Oiaga, Inequalities for certain submanifolds in complex space forms, Porc. 10th Int. Symposium Classical Analysis, Kazimierz Dolny (Poland), (1999), 49-61.

[23] A. Oiaga, I. Mihai, B.Y. Chen inequalities for slant submanifolds in complex space forms, Demonstratio Math., 32(4) (1999), 835-846.

[24] N. Papaghiuc, Semi-slant submanifolds of Kaehlerian manifold, Ann. St. Univ. Iasi, tom. XL, S.I., 9(f1) (1994), 55-61.

[25] F. Urbano, CR-submanifolds of nearly Kaehler manifolds, Doctoral Thesis, Granada (1980).

[26] K. Yano, M. Kon, Structures on manifolds, World Scientific, Singapore (1984).

1 Department of Mathematics, University of Allahabad, Allahabad, U.P., INDIA-211002

E-mail address: ssshukla_au@rediffmail.com

2 Department of Mathematics, University of Allahabad, Allahabad, U.P., INDIA-211002

E-mail address: babapawanrao@rediffmail.com 